\title{
Benchmarking Human Protein Complexes to Investigate Drug-Related Systems and Evaluate Predicted Protein Complexes
}

Min Wu, Qi Yu, Xiaoli Li, Jie Zheng, Jing-Fei Huang and Chee-Keong Kwoh

\section{A drug-complex network}

The drug-complex network for our CHPC2012 complexes consists of 2648 nodes, including 1835 drugs and 813 complexes, and 9916 edges as shown in the following Figure S1. In this figure, orange diamonds represent drugs and green circles are protein complexes in CHPC2012.

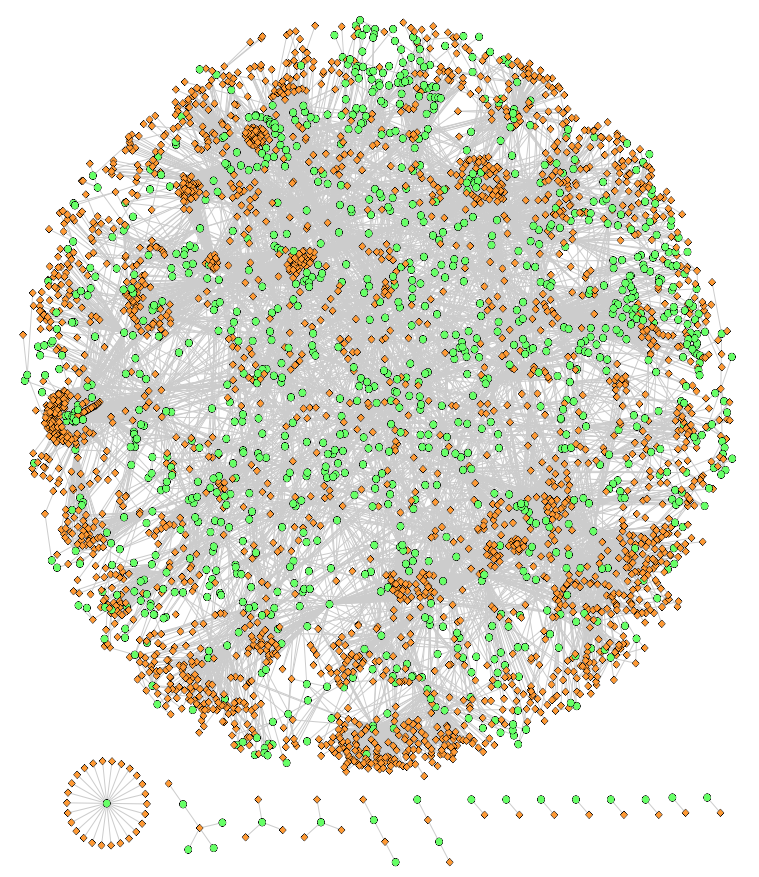

Fig. S1. The drug-complex network for complexes in CHPC2012. 


\section{Human protein complexes in Gene Ontology (GO)}

We processed the "cellular-component" sub-ontology of GO and collected 486 complexes for human. Table S1 shows the co-complex associations for these 486 complexes in GO, as well as three aforementioned databases, i.e., CORUM, HPRD and PINdb. We can find that the complexes in GO have the lowest fraction of co-complex protein associations in HPRD and BioGrid PPI databases. This indicates that the raw set of GO complexes has the lowest quality.

Table S1. Co-complex protein associations for 4 raw databases and their overlap with HPRD and BioGrid protein interactions.

\begin{tabular}{|c|c|c|c|c|}
\hline \hline Raw databases & CORUM & HPRD & PINdb & GO \\
\hline \# co-complex pairs & 35361 & 24214 & 6943 & 22990 \\
\hline Overlap with HPRD (\# pairs) & 2475 & 2843 & 614 & 1382 \\
\hline Overlap with HPRD (ratio) & $7.00 \%$ & $11.74 \%$ & $8.80 \%$ & $6.01 \%$ \\
\hline Overlap with BioGrid (\# pairs) & 4384 & 4044 & 1992 & 2000 \\
\hline Overlap with BioGrid (ratio) & $12.40 \%$ & $16.70 \%$ & $28.69 \%$ & $8.70 \%$ \\
\hline
\end{tabular}

We also processed the GO complexes with our Algorithm 1 (see the main manuscript). Table $\mathrm{S} 2$ shows that the quality of GO complexes is not improved in terms of the percentage of co-complex associations in existing PPI databases. However, the quality of complexes in other 3 databases (CORUM, HPRD and PINdb) is improved significantly as shown in Table S2. Note that CHPC2012 is obtained by integrating CORUM, HPRD and PINdb databases. The results in Tables S1 and S2 demonstrate that the quality of GO complexes is not good and this is the main reason we did not include GO complexes to build our CHPC2012.

Table S2. Co-complex protein associations for 5 processed databases and their overlap with HPRD and BioGrid protein interactions.

\begin{tabular}{|c|c|c|c|c|c|}
\hline \hline Processed databases & CHPC2012 & CORUM & HPRD & PINdb & GO \\
\hline \# co-complex pairs & 17810 & 10318 & 10592 & 4182 & 22819 \\
\hline Overlap with HPRD (\# pairs) & 3245 & 1925 & 2486 & 506 & 1375 \\
\hline Overlap with HPRD (ratio) & $18.22 \%$ & $18.66 \%$ & $23.47 \%$ & $12.10 \%$ & $6.02 \%$ \\
\hline Overlap with BioGrid (\# pairs) & 4853 & 3061 & 3356 & 1470 & 1994 \\
\hline Overlap with BioGrid (ratio) & $27.25 \%$ & $29.67 \%$ & $31.68 \%$ & $35.15 \%$ & $8.74 \%$ \\
\hline
\end{tabular}

\section{$3 \quad$ Parameter Settings}

In the Algorithm 1 in our main manuscript, there are two parameters namely overlap_thres and merge_thres. 
For the parameter overlap_thres, it is used to determine whether two complexes are redundant (i.e., they can match each other). In a previous study [1], this parameter is set as 0.5 . Therefore, we have also followed their suggestion and set it as 0.5 in our experiments. Let us give a specific example for this parameter. Suppose that there are two complexes and both of them have 8 proteins, they can be considered to be matching when the number of proteins in common between them is at least 6 (if the intersection has 6 proteins, the union will have 10 proteins and the Jaccard coefficient is 0.6 ; if the intersection has 5 proteins, the union will thus have 11 proteins and the Jaccard coefficient is 0.455 ) [1].

For the parameter merge_thres, it is used to determine whether to merge two redundant complexes or not. We are cautious to process those redundant complexes. If the value of the parameter merge_thres is set too low, then we may arbitrarily merge two different complexes as long as they share some protein components, which could generate false positive protein complexes.

As introduced in the main manuscript, co-complex protein associations are defined as all the pair-wise links between proteins within the same complexes. We can further assess the quality of a set of protein complexes by mapping its co-complex associations to existing PPI databases - A set of protein complexes that have higher percentage of co-complex associations overlapping with existing PPI databases tend to have higher quality [2].

As we know, CHPC2012 will have a different number of complexes when we use different values for merge_thres. Following your comments, we have performed additional experiments to investigate how the values of merge_thres affect the quality of final protein complex list. In particular, Figure S2 shows the percentage (ratio) of co-complex associations of CHPC2012 that overlaps with existing PPI databases, i.e., HPRD and BioGrid. The overall trend for the ratio curves is quite obvious - the ratio (i.e., the quality of CHPC2012) increases as the values of merge_thres incresase. However, we can still have the following two additional observations from Figure S2.

Firstly, the ratio increases quite rapidly as we increase the value for merge_thres when merge_thres is small (i.e., in [0.5, 0.65]). Smaller merge_thres leads to even lower quality of CHPC2012, indicating that the merging of complexes is indeed arbitrary using small values for merge_thres.

Secondly, the ratio increases much slowly when merge_thres is relatively large (i.e., in $[0.7,0.95])$. This indicates that the quality of CHPC2012 is stable and guaranteed after merge_thres becomes big. Therefore, we prefer to set merge_thres in the range [0.7, 0.95]. On the other hand, the number of co-complex associations and proteins covered by CHPC2012 decreases as we increase the value for merge_thres. This demonstrates that the coverage of CHPC2012 will decrease as merge_thres increases. To balance the coverage and the ratio of co-complex associations, we finally set merge_thres as 0.8 in our experiments. 


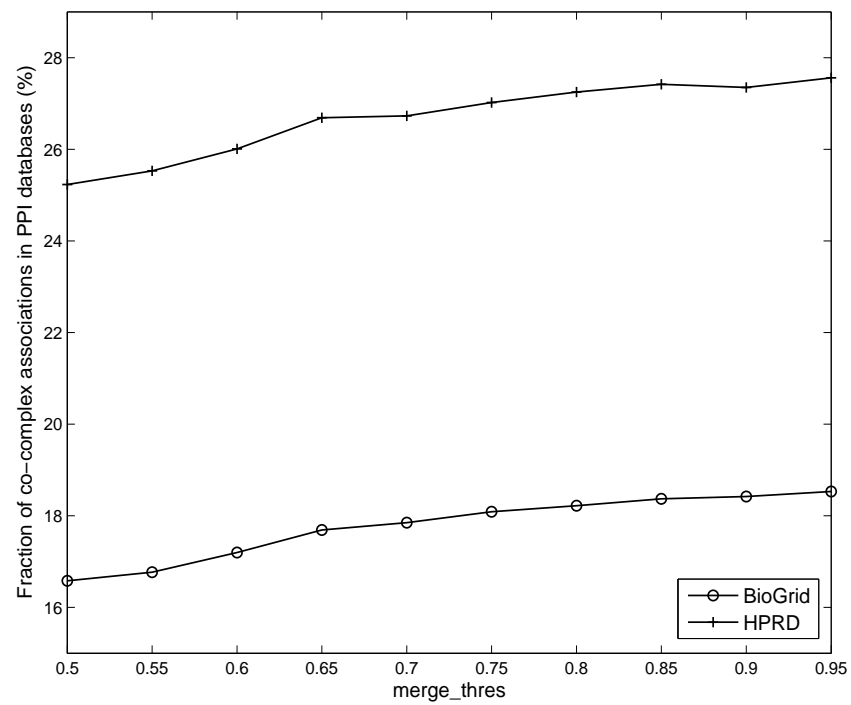

Fig. S2. The fraction of co-complexes associations in CHPC2012 overlapping in existing PPI databases, using different values for merge_thres.

Table S3. Details of CHPC2012 collected by using different values for the parameter merge_thres.

\begin{tabular}{|c|c|c|c|c|c|c|c|c|c|c|}
\hline \hline merge_thres & 0.5 & 0.55 & 0.6 & 0.65 & 0.7 & 0.75 & 0.8 & 0.85 & 0.9 & 0.95 \\
\hline \#complexes & 1507 & 1411 & 1406 & 1412 & 1407 & 1388 & 1389 & 1390 & 1390 & 1389 \\
\hline \#proteins & 3298 & 3152 & 3140 & 3105 & 3093 & 3066 & 3065 & 3040 & 3037 & 3033 \\
\hline \#overlapping proteins & 1556 & 1442 & 1428 & 1393 & 1378 & 1350 & 1346 & 1335 & 1336 & 1326 \\
\hline \#co-complex pairs & 22902 & 20829 & 20128 & 18978 & 18695 & 17987 & 17810 & 17405 & 17328 & 17066 \\
\hline Overlap with HPRD (\#pairs) & 3796 & 3493 & 3463 & 3357 & 3337 & 3253 & 3245 & 3198 & 3191 & 3162 \\
\hline Overlap with HPRD (ratio) & $16.58 \%$ & $16.77 \%$ & $17.20 \%$ & $17.69 \%$ & $17.85 \%$ & $18.09 \%$ & $18.22 \%$ & $18.37 \%$ & $18.42 \%$ & $18.53 \%$ \\
\hline Overlap with BioGrid(\#pairs) & 5779 & 5317 & 5235 & 5066 & 4997 & 4860 & 4853 & 4772 & 4740 & 4703 \\
\hline Overlap with BioGrid(ratio) & $25.23 \%$ & $25.53 \%$ & $26.01 \%$ & $26.69 \%$ & $26.73 \%$ & $27.02 \%$ & $27.25 \%$ & $27.42 \%$ & $27.35 \%$ & $27.56 \%$ \\
\hline
\end{tabular}

\section{References}

1. G. M. Liu, H. N. Chua, and L. Wong. Complex discovery from weighted ppi networks. Bioinformatics, 25(15):1891-1897, 2009.

2. S. Pu, J. Wong, B. Turner, E. Cho, and S. J. Wodar. Up-to-date catalogues of yeast protein complexes. Nucleic Acids Research, 37(3):825-831, 2009. 